\title{
ON THE ANHARMONIC POLARONIC MODEL
}

\author{
J. KONIOR \\ Institute of Physics, Jagiellonian University \\ Reymonta 4, 30-059 Kraków, Poland
}

\begin{abstract}
Anharmonic polaronic model is considered. The model consists of the electronic subsystem, described within the extended Hubbard model, coupled to anharmonic local phonons. By the canonical Holstein-Lang-Firsov transformation the electron-phonon interaction term is ruled out and, then, the effective electronic Hamiltonian is obtained by averaging over the ground state of the phononic subsystem. It is shown that anharmonicity introduces two main additional factors as compared with the harmonic case. First, the band narrowing factor is less rapidly decaying function of the electron-phonon interaction strength. Second, anharmonicity introduces further renormalization of the on- and intersite interaction between fermions.
\end{abstract}

PACS numbers: 71.38. $+\mathrm{i}, 74.20 .-\mathrm{z}, 33.10 . \mathrm{Cs}$

\section{Introduction}

Models consisting of correlated fermions interacting with phonons have been widely used to describe different phenomena in solid state physics. Among others one can mention the application to heavy fermion systems, polymers, and superconductivity. In such models fermions are typically described within the Hubbard model (simple or extended) and fermions are dispersionless and harmonic. There are strong indications that for some applications the anharmonicity could be an important factor. Here one should mention the high-temperature superconductors where there is a strong evidence that anharmonic phonons do play an important role [1].

In this paper the anharmonic polaronic model is considered. The model consists of the electronic subsystem, described within the extended Hubbard model, coupled to anharmonic local phonons. In Sec. 2 the effective polaronic Hamiltonian is derived as a result of eliminating the electron-phonon (e-ph) interaction and taking average over the ground state of the phononic subsystem. In Sec. 3 we investigate numerically the anharmonic corrections to the effective Hamiltonian. Finally, Sec. 4 concludes the paper. 


\section{Effective polaronic Hamiltonian} where

The total Hamiltonian of the system of interest $H=H_{\mathrm{e}}+H_{\mathrm{e}-\mathrm{ph}}+H_{\mathrm{ph}}$,

$$
\begin{aligned}
& H_{\mathrm{e}}=-\mu \sum_{i, s} n_{i, s}-\sum_{i, j, s} t_{i j} c_{i, s}^{\dagger} c_{j, s}+U \sum_{i} n_{i, \uparrow} n_{i, \downarrow}+\frac{1}{2} \sum_{i, j} V_{i j} n_{i} n_{j}, \\
& H_{\mathrm{e}-\mathrm{ph}}=g_{1} \sum_{i} n_{i}\left(b_{i}+b_{i}^{\dagger}\right)+g_{2} \sum_{i} n_{i}\left(b_{i+\delta}+b_{i+\delta}^{\dagger}\right), \\
& H_{\mathrm{ph}}=\omega_{0} \sum_{i}^{i}\left(b_{i}^{\dagger} b_{i}+1 / 2\right)+\sum_{i} V^{(1)}\left(x_{i}\right),
\end{aligned}
$$

are the electronic, e-ph interaction, and phononic parts of the Hamiltonian, respectively. In these formulae the summation over $i(i, j)$ runs over all sites (all pairs of sites) of a given lattice, the operator $c_{i, s}^{\dagger}\left(c_{i, s}\right)$ creates (destroys) a fermion with a spin $s$ at the site $i, n_{i, s}=c_{i, s}^{\dagger} c_{i, s}, s=\uparrow$ or $s=\downarrow$, and $n_{i}=n_{i, \uparrow}+n_{i, \downarrow} ; \mu$ is the chemical potential, $U$ is the Hubbard-Coulomb on-site repulsion, and $t_{i j}$ and $V_{i j}$ are the intersite hopping and Coulomb interaction matrix elements, respectively. The summation over $\delta$ runs over all $z$ nearest-neighbor (NN) sites of a given site $i$ and $b_{i}^{\dagger}\left(b_{i}\right)$ is the $i$-th site phonon creation (annihilation) operator. The fermions interact with on-site and NN intersite local phonons: $g_{1}$ and $g_{2}$ are the respective strengths of these interactions, $\omega_{0}$ is the frequency of harmonic phonons, and the local potentials $V^{(1)}\left(x_{i}\right)\left[x_{i}=\left(b_{i}+b_{i}^{\dagger}\right) / \sqrt{2}\right]$ are introduced in order to describe the anharmonic effects. For the potential $V^{(1)}(x)$ we choose

$$
V^{(1)}(x)=V_{1} \exp \left(-\gamma x^{2} / 2\right)
$$

where $V_{1}$ and $\gamma$ are parameters which determine the exact shape of the anharmonic deviations of the site potential.

The effective Hamiltonian for the electron subsystem, $H_{\mathrm{eff}}$, is obtained from $H$ by a two-step procedure which consists of the Lang-Firsov canonical transformation that eliminates the electron-phonon interaction term $H_{\mathrm{e}-\mathrm{ph}}[2,3]$ and the average over the phonon ground state wave function $[4,5]$. The result is

$$
\begin{aligned}
& H_{\mathrm{eff}}=-\left[\mu+\Delta \mu^{(1)}+\Delta \mu^{(2)}\right] \sum_{i, s} n_{i, s}+\left[U+\Delta U^{(1)}+\Delta U^{(2)}\right] \sum_{i} n_{i, \uparrow} n_{i, \downarrow} \\
& +\frac{1}{2} \sum_{i, j}\left[V_{i j}+\Delta V i j^{(1)}+\Delta V_{i j}^{(2)}\right] n_{i} n_{j}-\sum_{i, j, s} \hat{t}_{i j} c_{i, s}^{\dagger} c_{j, s}
\end{aligned}
$$

where $\hat{t}_{i j}=t_{i j} F\left(\alpha_{12}\right) F\left(-\alpha_{12}\right)\left[F\left(\alpha_{2}\right) F\left(-\alpha_{2}\right)\right]^{z-1}, \alpha_{12}=\sqrt{2}\left(g_{1}-g_{2}\right) / \omega_{0}, \alpha_{2}=$ $\sqrt{2} g_{2} / \omega_{0}$, and

$$
F(\alpha)=\int_{-\infty}^{\infty} \psi_{i}^{*}(x) \psi_{i}(x-\alpha) \mathrm{d} x .
$$

Here $\psi_{i}(x)$ is the $i$-th site phononic ground state wave function. Renormalizations $\Delta \mu^{(1)}, \Delta U^{(1)}$, and $\Delta V_{i j}^{(1)}$ are due to harmonic phonons whereas the contributions $\Delta \mu^{(2)}, \Delta U^{(2)}$, and $\Delta V_{i j}^{(2)}$ are induced by anharmonicity (see [5] for details).

\section{Numerical results}

In the actual calculations we fixed values $\omega_{0}=1, \gamma=5$, and performed the numerical analysis for $V_{1}=0$ (harmonic case), $V_{1} / \omega_{0}=2$ (moderate anharmonicity), and $V_{1} / \omega_{0}=5$ (strong anharmonicity). In Fig. 1 we plot $V(x)=$ 


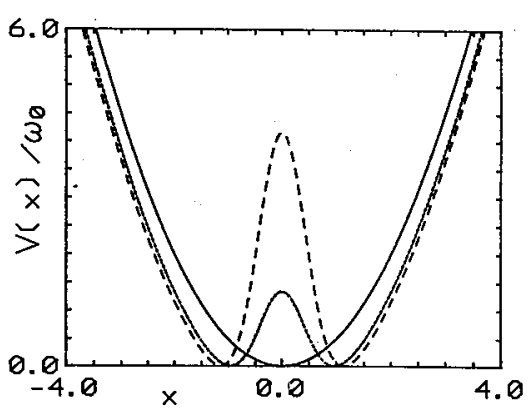

Fig. 1

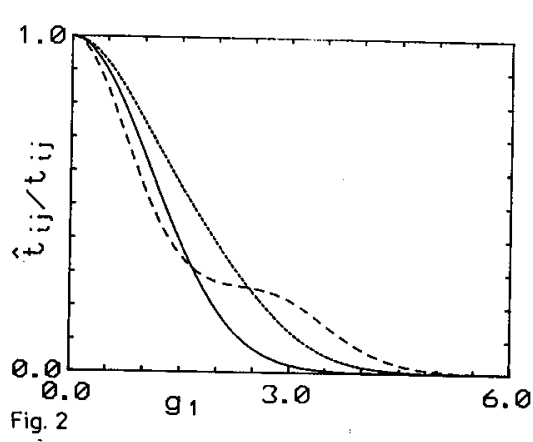

Fig. 1. Variation of the on-site ionic potential $V(x)$ with the distance $x$ for $\gamma=5$ and $V_{1}=0$ (solid line), $V_{1} / \omega_{0}=2$ (short dashed line), and $V_{1} / \omega_{0}=5$ (long dashed line); $\omega_{0}=1$ is the harmonic phonon frequency.

Fig. 2. The band narrowing factor $\hat{t}_{i j} / t_{i j}$ as a function of the on-site electron-phonon interaction strength $g_{1}$ and for $g_{2} / g_{1}=0.1 . g_{2}$ is the NN electron-phonon interaction strength. All parameters and labels are the same as in Fig. 1.

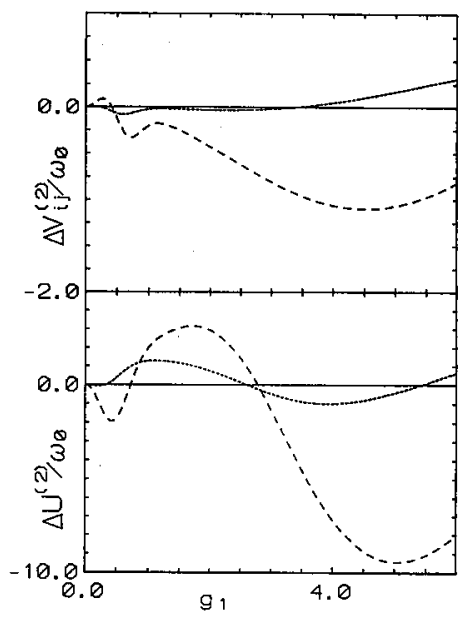

Fig. 3. Additional anharmonic renormalization of the on-site Hubbard interaction $U$ and the intersite Coulomb interaction $V_{i j}(i j$ are $N N), \Delta U^{(2)}$ and $\Delta V_{i j}^{(2)}$, respectively, as functions of the on-site electron-phonon interaction strength $g_{1}$ and for $g_{2} / g_{1}=0.1$. $g_{2}$ is the NN electron-phonon interaction strength. All parameters and labels are the same as in Fig. 1.

$\omega_{0} x^{2} / 2+V^{(1)}(x)$ for these values of $V_{1}$. For chosen values of $\gamma$ and $V_{1}$ we present values of $\hat{t}_{i j} / t_{i j}$ versus $g_{1}$ and for $g_{2} / g_{1}=0.1 \mathrm{in}$ Fig. 2 . Small values of $\hat{t}_{i j} / t_{i j}$ are equivalent to the high effective mass of polarons. From Fig. 2 we see that in the anharmonic polaronic model much higher values of the e-ph coupling $g_{1}$ are allowed without making the polarons too heavy. Figure 3 presents additional anharmonic 
renormalization of the on-site Hubbard-Coulomb and the intersite Coulomb interaction $\Delta U^{(2)}$ and $\Delta V_{i j}^{(2)}$, respectively. For the harmonic case $\Delta U^{(2)}=\Delta V_{i j}^{(2)}=0$. Up to moderate values of $V_{1}(\leq 2)$ these two corrections $\Delta U^{(2)}$ and $\Delta V_{i j}^{(2)}$ are rather small. Only for strong anharmonicity case (here $V_{1} / \omega_{0}=5$ ) these additional corrections seem to be significant provided the e-ph coupling $g_{1}$ is sufficiently large (larger than 3.0).

\section{Conclusions}

In this paper we have studied the anharmonic polaronic model in which the electronic subsystem, described by the extended Hubbard model, is coupled to the local anharmonic phonons. We have shown that, when going to the effective electronic Hamiltonian, the band narrowing factor is generally less rapidly decaying function of the e-ph coupling. It allows, at fixed value of the polaronic effective mass, for assuming higher values of the e-ph coupling without making the polarons too heavy. In addition to that, anharmonicity introduces additional renormalizations to the on-site and intersite interaction between fermions.

\section{Acknowledgments}

This work is a part of the Committee for Scientific Research project no. 2/0386/91/01.

\section{References}

[1] K.A. Müller, Phase Transit., special issue (1988); Z. Phys. B 80, 193 (1990).

[2] T. Holstein, Ann. Phys. 8, 325 (1959); I.G. Lang, Yu. A. Firsov, Sov. Phys.-JETP 16, 1301 (1963).

[3] A.N. Das, J. Konior, D.K. Ray, Physica C 170, 215 (1990).

[4] G.D. Mahan, Many Particle Physics, Plenum, New York 1981, Chap. 6.

[5] J. Konior, to be published. 